\title{
Derivation and Validation of a Clinical System for Predicting Pneumonia in Acute Stroke
}

\author{
Neale R. Chumbler ${ }^{a, b, e, p} \quad$ Linda S. Williams ${ }^{a, c, e, p} \quad$ Carolyn K. Wells $s^{f, g} \quad$ Albert C. Lo ${ }^{\text {h, j, } k}$ \\ Steven Nadeaul, m Aldo J. Peixoto ${ }^{g}$ Mark Gorman $^{n}$ John L. Boice ${ }^{o}$ John Concato ${ }^{c, f, i}$ \\ Dawn M. Bravata ${ }^{a}, \mathrm{~d}, \mathrm{e}, \mathrm{p}$

\begin{abstract}
aVA HSR\&D Center of Excellence on Implementing Evidence-Based Practice, Richard L. Roudebush VAMC, bepartment of Sociology, Indiana University School of Liberal Arts, Indiana University Purdue University Indianapolis, Departments of 'Neurology and ${ }^{\mathrm{d}}$ Internal Medicine, Indiana University School of Medicine, and eRegenstrief Institute, Indianapolis, Ind., ${ }^{f}$ Clinical Epidemiology Research Center and ${ }^{9}$ Medicine Service,

VA Connecticut Healthcare System, West Haven, Conn., Departments of heurology and 'Internal Medicine,

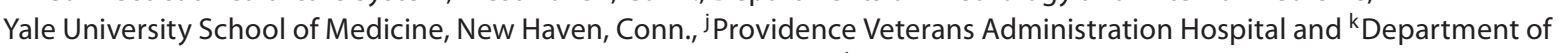
Neurology, Brown University School of Medicine, Providence, R.I., 'Neurology Service,

Malcolm Randall VAMC, and mDepartment of Neurology, University of Florida School of Medicine, Gainesville, Fla.,

${ }^{n}$ Department of Neurology, University of Vermont College of Medicine, Burlington, Vt., ${ }^{\circ}$ Medicine Service,
\end{abstract} \\ Boise VAMC, Boise, Idaho, and PVA HSR\&D Stroke Quality Enhancement Research Initiative Program, Indianapolis, Ind., USA
}

\section{Key Words}

Pneumonia $\cdot$ Stroke $\cdot$ Clinical prediction

\begin{abstract}
Aims: We derived and validated a clinical prediction rule that can be used to predict post-stroke pneumonia. Methods: We conducted a retrospective cohort study of patients admitted to hospital with a stroke. The cohort was subdivided into a derivation group and a validation group. Within the derivation group, a point scoring system was developed to predict pneumonia based on a logistic regression model. The point scoring system was then tested within the validation group. Results: Of the 1,363 patients with stroke, 10.5\% of patients experienced new pneumonia. The most points were assigned for abnormal swallowing result and history of pneumonia (4 points), followed by greater NIHSS score ( 3 points), patient being 'found down' at symptom onset (3 points), and age $>70$ years ( 2 points). A 3-level classifica-
\end{abstract}

tion system was created denoting low, medium and high risks of pneumonia, which accurately predicted pneumonia in the validation group. The discriminatory accuracy of the 3-level clinical prediction rule exceeded the acceptable range in both the derivation group (c statistic: 0.78 ) and validation group (c statistic: 0.76 ). Conclusion: A simple scoring system was derived and validated. This clinical scoring system may better identify stroke patients who are at high risk of developing new pneumonia.

Copyright $\odot 2010$ S. Karger AG, Basel

\section{Introduction}

Post-stroke pneumonia (newly developed pneumonia following stroke onset) is one of the most common serious adverse conditions among patients with ischemic stroke, with reported incidence rates ranging from 2.4 to $47 \%$, depending on type of treating unit and stroke sever-

\section{KARGER}

๑ 2010 S. Karger AG, Basel

Fax +4161306 1234 E-Mail karger@karger.ch www.karger.com www.karger.com/ned
Neale R. Chumbler, PhD

Richard L. Roudebush VAMC (11-H)

1481 W. 10th Street

Indianapolis, IN 46202 (USA)

Tel. +1 317988 3477, Fax +1 317988 3222, E-Mail neale.chumbler@va.gov 
ity of study population [1-3]. In spite of effective interventions for the prevention and treatment of pneumonia, post-stroke pneumonia is the leading cause of mortality after an acute stroke, accounting for nearly $35 \%$ of poststroke deaths [1, 4]. Moreover, post-stroke pneumonia is associated with a greater likelihood of being discharged to long-term care [5].

Previous research has identified various factors that are associated with post-stroke pneumonia, but no overall consistent pattern has emerged. Factors that have been associated with post-stroke pneumonia include: older age, diabetes, dysarthria/aphasia, cognitive impairment, stroke severity, and dysphagia $[4,6-10]$. Due to the variability in the research design of these post-stroke pneumonia studies (i.e. mixed acute and non-acute stroke patients, inclusion of non-stroke patients with stroke patients, limited number of factors under consideration), it has been difficult to identify a set of independent risk factors for post-stroke pneumonia. Identifying the stroke patients who are at the highest risk of pneumonia would allow clinicians to target resources and provide intensive prevention interventions, but no simple systems are currently available to identify patients at the greatest risk of post-stroke pneumonia.

The purpose of the present study was to develop and validate a clinical scoring system that would be simple to use and that could predict post-stroke pneumonia among patients with acute ischemic stroke.

\section{Methods}

\section{Study Participants}

This was a secondary analysis of a retrospective cohort study of patients admitted to 1 of 5 hospitals with a stroke (clinical syndrome differentiated by rapidly emerging clinical indications of global disturbance lasting $24 \mathrm{~h}$ or longer or leading to fatality with no apparent determinant other than of vascular origin) [11]. The hospitals, 3 Veterans Health Administration (VHA) and 2 nonVHA facilities, were selected to provide a varied group of settings in terms of: spectrum of stroke care (stroke neurology, general neurology, medicine); geographical diversity (Northeast: Connecticut; South: Florida; West: Idaho); and stroke patient volume (small, medium, and large). Cases were identified using the hospitals' administrative and clinical databases for the period 1/1/98 to $12 / 31 / 03$. Patients were included in the cohort if they had: an acute ischemic stroke; age $\geq 18$ years; neurological symptom onset within 2 days of hospital admission; and neurological deficit present at the time of admission (National Institutes of Health Stroke Scale, NIHSS, $\geq 2$ ). Patients were excluded if: they were residing in a skilled nursing facility at the time of the neurological symptom onset; they were admitted to the hospital prior to stroke symptom onset; and if they were transferred from a separate acute care facility. This study was approved by the institutional review board at Indiana University and the Research and Development Committee at the Roudebush Department of Veterans Affairs Medical Center in Indianapolis. Institutional review board approval was also attained at each of the individual VHA and nonVHA facilities.

\section{Outcome Variable}

The outcome was the clinical diagnosis of pneumonia made during the hospitalization for stroke. This outcome was measured by a single variable and classified as present or absent on the basis of any documentation of a clinical diagnosis of pneumonia in the medical record. We included only documentation made by a physician. Generally, this documentation was made in the assessment and plan and/or the problem list sections of the progress notes of the inpatient medical record.

\section{Independent Variables}

Several clinical and disease severity variables were assessed as potential risk factors in the clinical scoring system. These variables were selected because each factor was found to be associated with post-stroke pneumonia in previous studies. All of the independent variables (table 1) were measured at the time of admission to the hospital. The admission period was defined as the first $24 \mathrm{~h}$ after the time of admission. Demographic variables included patient sex, race/ethnicity (white, black, other) and age.

We included the following specific past medical history conditions: pneumonia, hypertension, diabetes, COPD, atrial fibrillation, and peptic ulcer disease. We also considered the Charlson co-morbidity index as an assessment of the patients' medical comorbidity (higher scores indicate severer co-morbidity) [12]. The patient's level of independence at home prior to stroke was measured by a binary variable (home without assistance vs. home with informal/formal assistance). Smoking status was coded as 'current smoker' versus 'other'. The presence or absence of a pre-existing swallowing problem was also recorded. Admission code status was classified as 'full code' versus other.

The baseline stroke severity was assessed using the NIHSS. The NIHSS was calculated retrospectively from the admission neurological examination recorded in the medical record [13]. The NIHSS was used as a continuous variable with higher scores indicating greater stroke severity. Stroke subtype was classified as a binary variable (lacune/lacunar syndrome vs. non-lacunar). We used a binary classification of stroke into lacunar versus non-lacunar because past evidence has indicated that there are no differences in risk factor profiles between lacunar and non-lacunar infarcts [14]. The patients were classified by the physicians' diagnostic category. Patients were considered to have a lacunar stroke if it was characterized by 1 of the following 4 categories: (1) pure motor hemiparesis (a unilateral motor deficit involving the face, the arm, and to a lesser extent the leg); (2) pure sensory stroke (characterized by unilateral numbness, paresthesias, and a hemisensory deficit involving the face, arm, trunk, and leg; (3) ataxic hemiparesis (weakness that is more prominent in the lower extremity in association with ipsilateral arm, lack of leg coordination, and dysarthria is not present); and (4) dysarthria-clumsy hand (characterized by supranuclear facial weakness, tongue deviation, dysphagia, dysarthria, impaired fine motor control of the hand, and an extensor plantar response).

We included 2 components of the stroke symptoms. The first component was a clinical presentation of whether or not the pa- 
Table 1. Characteristics of the patients

\begin{tabular}{|c|c|c|c|c|}
\hline & $\begin{array}{l}\text { Total sample } \\
(\mathrm{n}=1,363)\end{array}$ & $\begin{array}{l}\text { Derivation group } \\
(\mathrm{n}=925)\end{array}$ & $\begin{array}{l}\text { Validation group } \\
(\mathrm{n}=438)\end{array}$ & $\mathrm{p}$ \\
\hline \multicolumn{5}{|l|}{ Demographics } \\
\hline Male sex & $784(57.5)$ & $534(57.7)$ & $250(57.1)$ & 0.8201 \\
\hline \multicolumn{5}{|l|}{ Race } \\
\hline White & $1,070(78.5)$ & $724(78.3)$ & $346(79.0)$ & 0.7609 \\
\hline Non-White & $293(21.5)$ & $201(21.7)$ & $92(21.0)$ & \\
\hline Age (mean $\pm S D)$, years & $71.4 \pm 13.2$ & $71.5 \pm 13.3$ & $71.2 \pm 13.1$ & 0.6805 \\
\hline Age $\leq 70$ years & $537(39.4)$ & $362(39.1)$ & $175(40.0)$ & 0.7725 \\
\hline \multicolumn{5}{|l|}{ Past/Present Medical History } \\
\hline History of diabetes & $440(32.3)$ & $296(32.0)$ & $150(32.8)$ & 0.7465 \\
\hline History of hypertension & $983(72.1)$ & $666(72.0)$ & $317(72.4)$ & 0.8855 \\
\hline History of COPD & $179(13.1)$ & $136(14.7)$ & $43(9.8)$ & 0.0126 \\
\hline History of atrial fibrillation & $258(18.9)$ & $167(18.1)$ & $91(20.8)$ & 0.2309 \\
\hline History of peptic ulcer disease & $52(3.8)$ & $36(3.9)$ & $16(3.7)$ & 0.8297 \\
\hline History of pneumonia & $56(4.1)$ & $39(4.2)$ & $17(3.9)$ & 0.7711 \\
\hline Current smoker & $302(22.2)$ & $207(22.4)$ & $95(21.7)$ & 0.9627 \\
\hline Pre-existing swallowing problem & $20(1.5)$ & $13(1.4)$ & $7(1.6)$ & 0.0318 \\
\hline Charlson comorbidity score (mean \pm SD) & $3.0 \pm 2.4$ & $3.0 \pm 2.4$ & $2.9 \pm 2.3$ & 0.3216 \\
\hline \multicolumn{5}{|l|}{ Clinical Features } \\
\hline \multicolumn{5}{|l|}{ Stroke subtype } \\
\hline Lacunar & $231(16.7)$ & $160(17.3)$ & $78(17.0)$ & 0.6173 \\
\hline \multicolumn{5}{|l|}{ Level of independence prior to stroke } \\
\hline Independent (no help) & $1,086(79.7)$ & $733(79.2)$ & $368(80.5)$ & 0.6636 \\
\hline \multicolumn{5}{|l|}{ DNR status at admission } \\
\hline Full code & $1,238(91.0)$ & $834(90.3)$ & $412(90.2)$ & 0.4603 \\
\hline Abnormal swallowing test & $179(13.1)$ & $117(12.7)$ & $56(12.2)$ & 0.4419 \\
\hline Found down at symptom onset & $96(7.0)$ & $60(6.5)$ & $36(8.2)$ & 0.2430 \\
\hline Symptom course improving & $392(28.8)$ & $257(27.8)$ & $153(33.4)$ & 0.2472 \\
\hline \multicolumn{5}{|l|}{ Stroke Severity } \\
\hline Admission NIHSS score (mean \pm SD) & $9.4 \pm 7.3$ & $9.4 \pm 7.3$ & $9.6 \pm 7.7$ & 0.9224 \\
\hline Median & 7.0 & 7.0 & 7.0 & \\
\hline Interquartile range & 8.0 & 8.0 & 9.0 & \\
\hline \multicolumn{5}{|l|}{ Dependent Variable } \\
\hline New cases of pneumonia & $142(10.4)$ & $96(10.4)$ & $46(10.5)$ & 0.9443 \\
\hline
\end{tabular}

Figures in parentheses are percentages.

tient was 'found down' at symptom onset. 'Found down' refers to the extent to which, if a patient falls, he/she was unable to get up, and then someone finds the patient lying down usually (but not exclusively) on the ground. It is intended to capture the clinical implications of being down for a prolonged period of time. The second component was the course of the symptoms from the time of symptom onset to hospital admission (a nominal level variable classified as either 'stable', 'improving', 'worsening' or 'fluctuating'). 'Stable' refers to no change in symptoms; 'improving' captures the extent to which symptoms have gotten better; 'worsening' refers to steadily deteriorating symptoms; and 'fluctuating' depicts situations when symptoms occur, improve, and then worsen, or worsen and then improve (in this case 'improve' is taken to mean 'not resolved'). The variable was recoded so that 'stable', 'worsening' and 'fluctuating' were combined into a single category and labeled as 'not improving' versus 'improving'.

Among patients who received an assessment of swallowing function within $24 \mathrm{~h}$ of admission, dysphagia was defined on the basis of any abnormal swallowing test (e.g. fiberoptic examination, barium swallow, bedside examination). The bedside examination was a non-instrumented diagnostic test and consisted of having the patient swallow water and applesauce. In order for it to be coded as a bedside evaluation for our study, at least 2 modalities (e.g. water, applesauce, solids) needed to be tested. Dysphagia was categorized as present if there was dysphagia for liquids or solids or both. Dysphagia was categorized as absent if the swallowing study demonstrated normal swallowing function or other non-dysphagia results (e.g. slow mastication). 
Table 2. Predictors of pneumonia in the derivation group $(n=925)$

\begin{tabular}{llllr}
\hline & $\begin{array}{l}\text { Odds } \\
\text { ratio }\end{array}$ & $\begin{array}{l}\text { Points } \\
\text { allocated }\end{array}$ & 95\% CI & \multicolumn{1}{c}{$\mathrm{p}$} \\
\hline Patient age $>70$ years & 1.8 & 2 & $1.04-2.95$ & 0.0363 \\
Abnormal swallowing test & 3.5 & 4 & $2.08-5.73$ & $<0.0001$ \\
Admission NIHSS score & $3.1^{1}$ & 3 & $1.92-5.00$ & $<0.0001$ \\
Found down at symptom onset & 2.8 & 3 & $1.48-5.26$ & 0.0015 \\
Past medical history of pneumonia & 4.0 & 4 & $1.70-9.19$ & 0.0014 \\
\hline
\end{tabular}

Only significant $(\mathrm{p}<0.05)$ variables were included in this final regression model.

${ }^{1}$ This refers to a 3 -point per unit increase in the NIHSS score.
Statistical Analyses: Derivation of the Post-Stroke Pneumonia Prediction System

The total sample $(\mathrm{n}=1,363)$ was randomly separated into a derivation group (two thirds of total, $\mathrm{n}=925$ ) and validation group (one third of total, $n=438$ ). Statistical analyses were performed using a statistical package (SAS version 9.13, SAS Institute, Cary, N.C., USA).

The post-stroke pneumonia prediction system was developed only using the data from the derivation cohort. First, bivariate analyses ( $\chi^{2}$ test on discrete variables and tests on continuous variables) were employed to evaluate the association between each of the independent variables and the outcome of pneumonia.

Second, the variables that were associated with pneumonia in the bivariate analyses $(p<0.05)$ were entered into a multivariable logistic regression model. Backwards elimination was employed to select the final set of risk factors that were independently associated with pneumonia.

Third, we assigned points for each variable based on the adjusted odds ratios (AOR) from the multivariable model. The AORs were rounded to the nearest whole number to determine the points.

Fourth, a risk score was developed by summing the points for each risk factor present. Finally, we examined the outcome rate according to the risk score to identify 3 categories of risk: low, medium, and high risk of post-stroke pneumonia.

Statistical Analyses: Validation of the Post-Stroke Pneumonia Prediction System

The risk score and three category risk classification system were tested in the validation cohort. To assess the discriminatory ability of the pneumonia clinical scoring system, we calculated the $c$ statistic from a logistic regression model predicting pneumonia and including the variables identified from the development process [15]. The $c$ statistic, which represents the area under the receiver operating characteristic (ROC) curve, ranges from 0.5 (which indicates no better discrimination than chance) to 1.0 (perfect discrimination). A $c$ statistic value $>0.70$ is considered acceptable $[16,17]$. We compared the proportion of pneumonia cases as determined by the risk score with the actual rates of pneumonia (misclassification rate).

\section{Results}

Table 1 presents the patient characteristics of the total sample $(n=1,363)$, the derivation group $(n=925)$, and the validation group $(n=438)$. In the total sample, there were $142(10.4 \%)$ patients with pneumonia, with essentially identical proportions of pneumonia in the derivation $(10.4 \%)$ and validation (10.5\%) groups. In the total sample, $57.5 \%$ were male, $78.5 \%$ were White non-Hispanic, and the mean age was 71 years. Bivariate comparisons were performed to determine if statistically significant differences emerged between the derivation group and the validation group. A significant difference emerged between the 2 groups for history of COPD and pre-existing swallowing problem. None of the other variables differed between the 2 groups.

All of the variables in table 1 were associated with pneumonia in the bivariate analyses, and were therefore entered into the multivariate logistic regression model. Only 5 variables were independently associated with pneumonia (table 2): past medical history of pneumonia, dysphagia, increasing NIHSS (AOR for each unit of increase in NIHSS $3.10,95 \%$ CI 1.92-5.00), being found down at the time of symptom onset (AOR 2.79, 95\% CI $1.48-5.26$ ), and age $>70$ years [compared with age $\leq 70$ years (AOR 1.8, 95\% CI 1.04-2.95)]. The $c$ statistic from the development cohort denoted was $c=0.78$. The point scores for each of these 5 characteristics are presented in table 2 .

Table 3 presents the scoring algorithm for the 3 pneumonia risk categories for both the development and validation groups. The low-risk classification was based on a score of 0 (no risk factors present), the medium-risk classification was based on the presence of 1-3 risk factors, and the high-risk classification was based on the presence of 4 or more risk factors (table 3). In the development group, the pneumonia rate was $2 \%$ for the low-risk group, $4.2 \%$ for the medium-risk group, and $23 \%$ for the high- 
risk group. The $c$ statistic for the 3-category clinical prediction system in the development cohort was $c=0.78$. Similarly, among the validation group: the pneumonia rate was $0.90 \%$ for the low-risk group, $7.3 \%$ in the medium-risk group, and $21.5 \%$ in the high-risk group. The $c$ statistic for the 3-category clinical prediction system in the validation cohort was $c=0.76$. The misclassification rate for predicting pneumonia was $0.9 \%$ in the validation group and $2.1 \%$ in the derivation group (results not shown).

\section{Discussion}

The primary objective of the present study was to derive and validate a simple system that could be used to identify patients at highest risk of post-stroke pneumonia. Our results demonstrate that 5 patient characteristics (age $>70$ years, dysphagia, higher admission NIHSS, found down at symptom onset, and past medical history of pneumonia) can be used to identify patients at risk of post-stroke pneumonia.

The discriminatory accuracy of the 3-category clinical prediction system exceeded the acceptable range in both the derivation ( $c$ statistic: 0.78 ) and validation groups ( $c$ statistic: 0.76 ). The misclassification rate of our prediction rule was very low in both the derivation and validation group. Specifically, in the validation cohort, only 1 $(0.9 \%)$ of the 46 patients who had post-stroke pneumonia was misclassified as erroneously not having post-stroke pneumonia from the clinical scoring system. Therefore, the clinical usefulness of the 3-category clinical prediction may be realized both by classifying patients in the low-risk group (where the pneumonia rate in the validation cohort was less than 1\%) and by classifying patients in the high-risk group (where 1 in 5 patients in the validation cohort developed post-stroke pneumonia). This system may therefore be used both to identify patients who may not require intensive resources and those patients who are most likely to benefit from pneumonia prevention strategies.

Our study results were similar to the findings of Sellars et al. [6] who evaluated 412 acute stroke patients in the United Kingdom. They identified 5 variables which were associated with self-reported pneumonia: age $>65$ years, dysarthria or no speech due to aphasia, modified Rankin score $\geq 4$ [18], Abbreviated Mental Test [19], and failed staged water swallowing test that consisted of progressively larger amounts of water [20]. Even though these findings were informative, some of the factors as-
Table 3. Post-stroke pneumonia rates according to risk classification

\begin{tabular}{|c|c|c|c|c|c|}
\hline & \multirow{2}{*}{$\begin{array}{l}\text { Total } \\
\text { score }\end{array}$} & \multicolumn{2}{|c|}{ Derivation } & \multicolumn{2}{|c|}{ Validation } \\
\hline & & $\mathrm{n}$ & $\begin{array}{l}\text { pneumonia } \\
\text { rate, } \%\end{array}$ & $\mathrm{n}$ & $\begin{array}{l}\text { pneumonia } \\
\text { rate, \% }\end{array}$ \\
\hline Low & 0 & 239 & 2.1 & 111 & 0.90 \\
\hline Medium & $1-3$ & 354 & 4.2 & 178 & 7.3 \\
\hline High & $\geq 4$ & 332 & 22.9 & 149 & 21.5 \\
\hline Total & & 935 & & 438 & \\
\hline
\end{tabular}

Total scores of 0 (low risk), 1-3 (medium risk), and $\geq 4$ (high risk) indicate $0,1-3$, and $\geq 4$ risk factors present, respectively.

sociated with post-stroke pneumonia were based on testing that may not be routinely performed in clinical practice (i.e. the Abbreviated Mental Test or the staged water swallowing test).

Consistent with previous research, we found that older age, abnormal swallowing test, and worse stroke severity were associated with post-stroke pneumonia $[4,6$, 21-23]. However, we also identified 2 characteristics not previously reported to be predictive of post-stroke pneumonia - past medical history of pneumonia and 'found down' at symptom onset. Being 'found down' is rarely considered in the context of post-stroke pneumonia. Patients who are 'found down' at stroke onset may have aspirated gastric contents and actually have pneumonia present at the time of presentation to the hospital; however, this pneumonia may have been unrecognized until some point after admission. Similarly, patients with a history of pneumonia may either have been at increased risk of post-stroke pneumonia, or might have had unrecognized pneumonia present at admission.

Numerous studies have demonstrated that dysphagia is associated with post-stroke pneumonia, as well as poststroke mortality, persistent disability, and prolonged hospital stay $[6,24,25]$. Unlike the other (non-modifiable) factors that are associated with post-stroke pneumonia (e.g. patient age), swallowing problems can be treated. Several studies have demonstrated that the implementation of a dysphagia screening protocol can decrease the incidence of pneumonia in hospitalized acute stroke patients $[4,26,27]$. Although controversy exists regarding the most valid and suitable dysphagia screening assessment for stroke patients, the evidence clearly demonstrates that stroke patients should receive some assessment of swallowing function and that pneumonia pre- 
vention strategies should be implemented for patients with impaired swallowing.

The NIHSS is increasingly being used as the measure of stroke severity among patients with ischemic stroke. Our findings underscore the importance of using the NIHSS not only as a measure of stroke severity, but also as part of an assessment for pneumonia risk.

There were several strengths to this study, including the large sample, the geographically diverse location of patients recruited from both private and federal government hospitals, and the use of well-accepted and widely available demographic and clinical variables used for development of this clinical risk prediction system. However, the study had some limitations that should be acknowledged. First, these data were obtained from patients who were admitted to a hospital during 1998-2003. Despite this, we do not believe that clinical practice has changed substantially or that the associations between the demographic and clinical factors predictive of pneumonia would differ currently.

Second, given that the original sample excluded patients admitted from a skilled nursing facility and those patients who had a stroke 'in house,' these findings may not be generalizable to these patient groups. Since the majority of patients admitted with a stroke are coming from home, not from a nursing home or another hospital, our findings should be generalizable to the majority of stroke patients. Future studies should specifically address the issue of pneumonia risk prediction among nursing home patients who may be at higher risk of pneumonia than patients who have their stroke onset while residing at home.

Third, due to the nature of the retrospective chart review in our present study, our definition of pneumonia required documentation by the clinician. A verification of this clinical diagnosis via chest radiographs, cultures, and chest computed tomography was beyond the scope of the present study. However, this study may have underrecognized the true rates of post-stroke pneumonia overall and within risk categories. Future studies should test the validity of the 3 -category risk prediction system in a prospective cohort where pneumonia events are clearly defined and prospectively identified. Fourth, there is a possible confounding effect of parameters not taken into account in the present models. For instance, the patient's past or present medical history or concomitant medication, such as antibiotics at admission or immunosuppressive drugs, could lead to an immunosuppressive state.

\section{Conclusion}

We conclude that a simple 3-category clinical prediction system that consists of 5 variables (dysphagia, history of pneumonia, higher NIHSS score, patient 'found down' and age $>70$ years) can identify stroke patients at lower and higher risk of pneumonia. This clinical scoring system may be particularly relevant for hospitals using information technology systems. The clinical prediction system could easily be implemented and tested as part of a stroke decision support tool to prompt recognition of patients who are at high risk of developing pneumonia.

\section{Acknowledgments}

This study was supported by the Department of Veterans Affairs Health Services Research and Development Service (Merit IIR-01-104-3) and the Max Patterson Stroke Research Fund at Yale University. This study was also supported by resources from the Department of Veterans Affairs HSR\&D Center for Implementing Evidence-Based Practice, Richard L. Roudebush VAMC, Indianapolis, Ind., USA.

\section{References}

1 Walter U, Knoblich R, Steinhagen V, Donat M, Benecke R, Kloth A: Predictors of pneumonia in acute stroke patients admitted to a neurological intensive care unit. J Neurol 2007;254:1323-1329.

-2 Marciniak C, Korutz AW, Roth E, Welty L, Lovell L: Examination of selected clinical factors and medication use as risk factors for pneumonia during stroke rehabilitation. Am J Phys Med Rehabil 2009;88:30-38.

-3 Teramoto S: Novel preventive and therapeutic strategy for post-stroke pneumonia. Expert Rev Neurother 2009;9:1187-1200.
4 Hinchey JA, Shephard T, Furie K, Smith D, Wang D, Tonn S: Formal dysphagia screening protocols prevent pneumonia. Stroke 2005;36:1972-1976

5 Kammersgaard LP, Jorgensen HS, Rungby JA, Reith J, Nakayama H, Weber UJ, Houth J, Olsen TS: Admission body temperature predicts long-term mortality after acute stroke: The Copenhagen Stroke Study. Stroke 2002;33:1759-1762.
6 Sellars C, Bowie L, Bagg J, Sweeney MP, Miller H, Tilston J, Langhorne P, Stott DJ: Risk factors for chest infection in acute stroke: a prospective cohort study. Stroke 2007;38: 2284-2291.

7 Aslanyan S, Weir, Deiner HC, Kaste M, Lees KR, Gain International Steering Committee and Investigators: Pneumonia and urinary tract infection after acute ischemic stroke: a tertiary analysis of the GAIN International Trial. Eur J Neurol 2004;11:49-53. 
8 Martino R, Foley N, Bhogal S, Diamant N, Speechley M, Teasell R: Dysphagia after stroke: incidence, diagnosis, and pulmonary complications. Stroke 2005;36:2756-2763.

$>9$ Ding R, Logemann JA: Pneumonia in stroke patients: a retrospective study. Dysphagia 2001;15:51-57.

- 10 Mann G, Dip PG, Hankey GJ, Cameron D: Swallowing function after stroke: prognosis and prognostic factors at 6 months. Stroke 1999;30:744-748.

11 Liebeskind DS: Case fatality rates after hospital admission for stroke: definition of stroke defines stroke mortality. BMJ 2003; 326:1085-1086.

12 Charlson M, Pompei P, Ales K, MacKenzie C: A new method of classifying prognostic comorbidity in longitudinal studies: development and validation. J Chron Dis 1987;40: 373-383.

$\checkmark 13$ Williams LS, Yilmaz EY, Lopez-Yunez AM: Retrospective assessment of initial stroke severity with the NIH Stroke Scale. Stroke 2000;31:858-862.

14 Jackson C, Sudlow C: Are lacunar strokes really different? A systematic review of differences in risk factor profiles between lacunar and nonlacunar infarcts. Stroke 2005;36: 891-901.
15 Hanley JA, McNeil BJ: The meaning and use of the area under the receiver operating characteristic (ROC) curve. Radiology 1982;43: 29-36.

16 Hosmer DH, Lemeshow S: Applied Logistic Regression. New York, Wiley, 2000.

-17 Janssen I, Katzmarzyk PT, Church TS, Blair SN: The Cooper Clinic Mortality Risk Index clinical score sheet for men. Am J Prev Med 2005;29:194-203.

18 van Swieten JC, Koudstaal PJ, Visser MC, Schouten HJ, van Gijn J: Interobserver agreement for the assessment of handicap in stroke patients. Stroke 1988;19:604-607.

-19 Hodkinson HM: Evaluation of a mental test score for assessment of mental impairment in the elderly. Age Ageing 1972;1:233-238.

20 Perry L, Love CP: Screening for dysphagia and aspiration in acute stroke: a systematic review. Dysphagia 2001;16:7-18.

21 Hilker R, Poetter C, Findeisen N, Sobesky J, Jacobs A, Neveling M, Heiss WD: Nosocomial pneumonia after acute stroke: implications for neurological intensive care medicine. Stroke 2003;34:975-981.
22 Upadya A, Thorevska N, Sena KN, Manthous C, Amoateng-Adjepong Y: Predictors and consequences of pneumonia in critically ill patients with stroke. J Crit Care 2004;19: 16-22.

23 Dziewas R, Ritter M, Schilling M, Konrad C, Oelenberg S, Nabavi DG, Stögbauer F, Ringelstein EB, Lüdemann P: Pneumonia in acute stroke patients fed by nasogastric tube. J Neurol Neurosurg Psychiatry 2004;75:852856.

24 Ramsey DJ, Smithard DG, Kalra L: Early assessments of dysphagia and aspiration risk in acute stroke patients. Stroke 2003;34:12521257.

25 Smithard DG, Smeeton NC, Wolfe CD: Long-term outcome after stroke: does dysphagia matter? Age Ageing 2007;36:90-94.

$>26$ Odderson IR, Keaton JC, McKenna BS: Swallow management in patients on an acute stroke pathway: quality is cost effective. Arch Phys Med Rehabil 1995:76:1130-1133.

$>27$ Doggett DL, Tappe KA, Mitchell MD, Chapell R, Coates V, Turkelson CM: Prevention of pneumonia in elderly stroke patients by systematic diagnosis and treatment of dysphagia: an evidence-based comprehensive analysis of the literature. Dysphagia 2001;16: 279-295. 